\title{
Evaluación del sistema de salud mental en México: ¿hacia dónde encaminar la atención?
}

\author{
Shoshana Berenzon Gorn, ${ }^{1}$ Nayelhi Saavedra Solano, ${ }^{1}$ \\ María Elena Medina-Mora Icaza, ${ }^{1}$ Víctor Aparicio Basaurí ${ }^{2}$ \\ y Jorge Galván Reyes ${ }^{1}$
}

Forma de citar

Berenzon Gorn S, Saavedra Solano N, Medina-Mora Icaza ME, Aparicio Basaurí V, Galván Reyes J. Evaluación del sistema de salud mental en México: ¿hacia dónde encaminar la atención? Rev. Panam Salud Publica. 2013;33(4):252-8.

RESUMEN Objetivo. Evaluar algunos indicadores clave del sistema de salud mental mexicano utilizando el Instrumento de Evaluación para Sistemas de Salud Mental de la Organización Mundial de la Salud (IESM-OMS).

Métodos. La estrategia utilizada para responder a los indicadores del IESM-OMS incluyó: i) Revisión de fuentes documentales, ii) Aplicación de cuestionarios y iii) Trabajo grupal utilizando la técnica de consenso con expertos. Para facilitar la recopilación de la información, se elaboró un cuestionario donde los indicadores fueron transformados en preguntas sencillas. Asimismo, se capacitó a las personas encargadas de recabar los datos y se realizó un seguimiento de dicha actividad.

Resultados. Del total del presupuesto asignado a la salud, solo $2 \%$ estaba destinado a la salud mental $y$, de ese porcentaje, $80 \%$ se empleaba para el funcionamiento de los hospitales psiquiátricos. El eje de la atención de la salud mental se encontraba en el hospital psiquiátrico y existían muy pocas unidades de hospitalización psiquiátrica en los hospitales generales y establecimientos residenciales, así como servicios específicos para niños y adolescentes. El acceso estaba limitado por la centralización del sistema de atención, ya que la mayoría de los establecimientos se ubicaban en las grandes ciudades. Solamente $30 \%$ de los servicios de atención primaria contaban con protocolos de evaluación y tratamiento para trastornos mentales. Finalmente, en los establecimientos de salud mental, la tasa de psiquiatras, médicos, enfermeros y psicólogos por cada 100000 habitantes registró valores de 1,6, 1,3, 3,4 y 1,5 respectivamente.

Conclusiones. Para reducir la brecha entre la carga que representan los trastornos mentales y el presupuesto asignado para su atención, se requiere de un mayor financiamiento y una utilización de los recursos más racional, considerando al primer nivel como el eje de la atención. Asimismo, será necesario ampliar el número de especialistas, capacitar periódicamente al personal en los primeros niveles de atención e incrementar la participación del resto de la sociedad.

Palabras clave Salud mental; formulación de políticas; hospitales psiquiátricos; trastornos mentales; servicios de salud mental; México.

1 Instituto Nacional de Psiquiatría Ramón de la Fuente Muñiz, México D.F., México. La correspondencia se debe dirigir a Shoshana Berenzon Gorn. Correo electrónico: berenz@imp.edu.mx

2 Hospital Universitario Central de Asturias, Principado de Asturias, España.
La salud mental representa desde hace tiempo una cuenta pendiente para la salud pública de muchos países del mundo, y ha quedado siempre relegada frente a otros padecimientos que las po- líticas públicas consideran prioritarios. Por esa razón los recursos invertidos en salud mental suelen ser pocos e insuficientes. En las Américas, en promedio solo $1,53 \%$ del gasto total en salud está 
destinado a salud mental $(1,2)$. Frente a esta situación, y con el propósito de animar a los países en desarrollo a revisar sus políticas públicas y establecer mecanismos que proporcionen datos confiables, la Organización Mundial de la Salud (OMS) desarrolló el Instrumento de Evaluación para Sistemas de Salud Mental (IESM-OMS), el cual recopila información clave para evaluar y fortalecer los servicios de salud mental en todo el mundo $(3,4)$. El IESM-OMS fue implementado en México entre 2009 y 2010, con el objetivo de tener un conocimiento más acabado de la estructura y la distribución de los recursos de atención en materia de salud mental.

México posee una gran diversidad cultural, social y económica, su población en 2010 era de 112336538 habitantes y está compuesto por 31 estados y un Distrito Federal. A pesar de estar considerado por el Banco Mundial como un país con un nivel de ingreso medio-alto, la inequidad -expresada en importantes falencias en aspectos clave como salud, educación, alimentación y servicios básicos- continúa siendo una de sus principales dificultades $(5,6)$. Sumado a esto, en la actualidad atraviesa un momento difícil, donde la inseguridad y la violencia son dos grandes preocupaciones de la población mexicana, razón por la cual el miedo y la desesperanza se han vuelto moneda corriente entre sus habitantes.

Al igual que ocurriera en muchos otros países de América Latina, México ha atravesado un conjunto de severas crisis económicas que derivaron en una reducción paulatina del gasto público en salud $-\mathrm{y}$, consecuentemente, del gasto en salud mental. Las respuestas - de los sucesivos gobiernos - frente a esta situación trajeron aparejada una progresiva descentralización de los servicios proporcionados por el Estado, en un intento de ampliar las coberturas, aprovechar los recursos con mayor racionalidad y obtener la mejor relación costo-beneficio en las prestaciones públicas. Tal descentralización incluyó la transferencia de los programas federales a los servicios de los estados para asegurar su normal funcionamiento (7).

Los modelos asistenciales implementados priorizaron claramente la sanación - por sobre la promoción y la prevención de la salud-, y se hicieron efectivos a través de paquetes de servicios básicos que garantizan un número definido de acciones en salud. Un ejemplo de ello es el Seguro Popular, ${ }^{3}$ que si bien ha reducido de manera considerable algunas demandas de atención en salud, resulta insuficiente en su cobertura para el campo de la salud mental (8-10).

Hoy por hoy México experimenta una transición epidemiológica "polarizada", cuyos rasgos más notorios son la disminución de enfermedades infectocontagiosas y el aumento de padecimientos crónico-degenerativos, incluidos los trastornos mentales que constituyen un problema importante de salud pública debido a su elevada prevalencia, la gran carga de enfermedad que generan y los altos costos económicos y sociales que producen $(1,11)$.

A nivel mundial, se ha estimado que las tasas de prevalencia de trastornos mentales en el curso de la vida oscilan entre $12,2 \%$ y $48,6 \%$, mientras que la prevalencia anual rondaría entre $8,4 \%$ y $29,1 \%$. El 14\% de la carga de morbilidad en todo el mundo, medida en años de vida ajustados en función de la discapacidad (AVAD), es atribuible a este tipo de padecimientos y es aún más patente en los países de bajos y medianos ingresos (12). En 1990 los trastornos neuropsiquiátricos representaban un $8,8 \%$ de los AVAD de América Latina y el Caribe, cifra que aumentaría a 22,2\% en 2002 (13).

Uno de cada cuatro mexicanos de entre 18 y 65 años ha padecido en algún momento de su vida un trastorno mental, pero solo uno de cada cinco de los que lo padecen recibe tratamiento. El tiempo que demoran los pacientes para recibir atención en un centro de salud oscila entre 4 y 20 años según el tipo de afección, al igual que lo observado en la mayoría de los países de América Latina (14-18).

Resultados de evaluaciones realizadas con el IESM-OMS en América Central y República Dominicana señalan que la mayor parte de estos países presentan importantes limitaciones en sus sistemas de salud mental, en particular la falta de recursos financieros y humanos, las deficiencias en la protección de los derechos

\footnotetext{
3 Desde 2004, la población que no se encontraba incluida dentro de la cobertura de la seguridad social ( $45 \%$ de la población mexicana) comienza a recibir atención mediante el Seguro Popular. El seguro fue ampliando su cobertura de manera escalonada y el financiamiento del sistema incluyó una cuota social proveniente de aportaciones federales y estatales, y otra privada que corresponde al afiliado de acuerdo a su condición socioeconómica. En 2010 se incluyó en el seguro la atención ambulatoria y la hospitalización breve para los principales trastornos mentales (7).
}

relacionados con la salud, el desarrollo insuficiente del componente salud mental en el primer nivel de atención y la escasa participación de la sociedad civil (19).

En este contexto, el propósito del presente trabajo fue precisamente evaluar algunos indicadores clave del sistema de salud mental mexicano utilizando el IESM-OMS.

\section{MATERIALES Y MÉTODOS}

\section{Instrumento}

El IESM-OMS está compuesto por seis secciones:

1. Política y marco legislativo. Contiene indicadores de los componentes incluidos en los planes, políticas y leyes relacionados con la salud mental. Indaga también sobre los gastos y fuentes financieras de estos servicios, así como respecto de las funciones de los organismos encargados de proteger los derechos humanos de los pacientes y sus familiares.

2. Servicios de salud mental. Aborda las características específicas de cada uno de los servicios de salud, tales como establecimientos ambulatorios, establecimientos de tratamiento diurno, unidades de hospitalización dentro de hospitales generales, establecimientos residenciales, hospitales psiquiátricos y unidades forenses. Se pregunta además sobre la disposición de medicamentos psicotrópicos e intervenciones psicosociales.

3. Salud mental en la atención primaria. Busca conocer cuál es la formación que tienen los médicos de la atención primaria en temas relacionados con la salud mental, su vinculación con los servicios especializados en salud mental y su interacción con profesionales dedicados a las medicinas alternativas/tradicionales. ${ }^{4}$ La interacción incluye reuniones, revisión de casos, coordinación de actividades y asuntos de referencia a pacientes, así como la formación de profesionales alternativos/tradicionales en temas relacionados con la salud mental.

\footnotetext{
4 Un conjunto diverso de sistemas, prácticas y productos médicos y de atención a la salud que no se consideran dentro de la medicina oficial/formal. Por ejemplo, el curanderismo, la acupuntura y la aromaterapia.
} 
4. Recursos humanos. Incluye una serie de indicadores sobre el personal que trabaja en servicios de salud mental y su proceso de formación, así como un conjunto de preguntas para evaluar las características de las asociaciones de usuarios y familiares.

5. Educación sanitaria y vínculos con otros sectores. Examina la existencia de campañas de educación y concientización a la población en temas de salud mental y la vinculación con sectores como el educativo, el laboral o el judicial.

6. Monitoreo y evaluación. La primera parte — de la sección - busca conocer las características de la recopilación e informes rutinarios de datos claves en establecimientos de salud mental y, la segunda, la extensión y el contenido de la investigación en este campo.

El IESM-OMS fue confeccionado a partir de las 10 recomendaciones del Informe mundial de la Salud de 2001, y las secciones que lo componen están organizadas en 28 apartados y 156 indicadores (20). A fin de evaluar la claridad y viabilidad de la aplicación de este instrumento, se realizaron ensayos en 12 países de bajos recursos económicos -y en 2005 se publica su versión final. Desde esa fecha, 78 países han utilizado el IESM-OMS para analizar sus sistemas de salud mental $(1,4,21)$.

\section{Recolección de la información}

Con el propósito de responder a los 156 indicadores del IESM-OMS, se realizó la recopilación de la información de las tres formas siguientes: (i) revisión de fuentes documentales, como bases de datos nacionales, anuarios estadísticos, normas y leyes oficiales; (ii) administración de un cuestionario en los diversos servicios de salud mental ubicados en cada uno de los estados de México y (iii) realización de un trabajo grupal con expertos en el área de la salud mental. Toda la información obtenida corresponde al año 2008.

\section{Procedimiento}

Aplicación del cuestionario. Para facilitar la tarea de recopilar la información, se elaboró un cuestionario donde cada uno de los indicadores propuestos —del IESM-OMS - se transformó en una pregunta sencilla. Por ejemplo, el ítem 2.2.1 —número de establecimientos de salud mental ambulatorios- fue llevado a la fórmula “¿Cuántos establecimientos de salud mental ambulatorios hay en su estado?". El cuestionario fue revisado por un experto en salud mental, responsable de la supervisión de la aplicación del IESM-OMS en América Latina y el Caribe.

Asimismo, se asignó una persona encargada de recabar la información sobre las características de los servicios de salud mental en su entidad para cada uno de los 32 estados del país. Los encargados recibieron un curso de capacitación que explicaba en detalle las características del instrumento y buscaba homogeneizar las estrategias de recopilación. Del año 2009 a 2010 se llevó a cabo el trabajo de recopilación, período en el que se mantuvo un contacto estrecho con los encargados estatales - para brindarles apoyo técnico- y se hizo el seguimiento de la recopilación.

Técnica de consenso. Para recabar información sobre aquellas preguntas del IESM-OMS que necesitaban una valoración o estimación se utilizó la técnica de consenso, a través de la cual se busca obtener un acuerdo entre diversos especialistas en la materia. Con ese objetivo se invitó a un conjunto de expertos en el área de salud mental de cada uno de los estados del país y se les entregó un cuestionario individual. Posteriormente, se expusieron las ideas generadas en los cuestionarios en una reunión de consenso, donde fueron discutidas y sometidas a votación para terminar de definir las respuestas (22).

Análisis de la información. Los datos obtenidos de las diferentes fuentes fueron capturados y procesados mediante el Programa de Ingreso de Datos de Excel del IESM-OMS (23). Este software permite realizar análisis descriptivos de forma sencilla y sin margen para errores de cálculo, impidiendo además que se capturen datos que están fuera de rango - por ejemplo, un mayor número de pacientes ambulatorios que contactos ambulatorios.

La preparación de los cuestionarios, la capacitación impartida, el apoyo técnico brindado, la validación de los resultados y la elaboración de las bases de datos estatal y nacional, así como la redacción del informe final, estuvieron a cargo del personal del Instituto Nacional de Psiquiatría Ramón de la Fuente Muñiz (México D.F.) y la Organización Panamericana de la Salud/OMS.

\section{RESULTADOS}

\section{Política, marco legislativo y financiamiento}

Desde hace más de dos décadas, México dispone de leyes, normas y programas de acción que regulan la atención de la salud mental e incluyen los lineamientos básicos para las políticas públicas destinadas a paliar este tipo de afecciones. Se cuenta con un órgano independiente del sector salud, la Comisión Nacional de los Derechos Humanos, encargada de controlar que se respeten los derechos humanos de los usuarios y familiares. El $67 \%$ de los hospitales psiquiátricos del país recibieron en 2008 por lo menos una revisión/inspección sobre derechos humanos y $63 \%$ del personal que trabaja en esos hospitales ha recibido algún tipo de capacitación sobre este tema. Sin embargo, las acciones de inspección solamente se realizaron en $14 \%$ de las unidades de hospitalización psiquiátrica de los hospitales generales y establecimientos residenciales comunitarios, y solo una tercera parte del personal de dichos establecimientos había recibido alguna capacitación relacionada con el tema.

Como se observa en el cuadro 1, si bien en México el porcentaje del presupuesto de salud asignado a salud mental entre 2005 y 2009 fue superior a la media de los países de las Américas, continuaba siendo inferior al destinado en otros países con economías similares como Costa Rica o Panamá (19). Además, el 80\% del presupuesto de salud mental era utilizado para la operación de los hospitales psiquiátricos —dejando solo $20 \%$ para financiar al resto de la red de servicios de salud mental-, lo que reducía, por ejemplo, la capacidad de acciones de salud mental comunitaria.

Por último, se encontró también que el gobierno subvencionaba el costo de los medicamentos psicotrópicos para $85 \%$ de la población que requería de estos fármacos, mientras que el $25 \%$ restante 
CUADRO 1. Presupuesto destinado a salud mental (SM) y a hospitales psiquiátricos (HP) en países seleccionados de América Latina, 2005-2009

\begin{tabular}{lcc}
\hline \multicolumn{1}{c}{ País } & $\begin{array}{c}\text { Presupuesto de salud } \\
\text { destinado a SM (\%) }\end{array}$ & $\begin{array}{c}\text { Presupuesto de SM } \\
\text { destinado a HP (\%) }\end{array}$ \\
\hline Costa Rica & 2,9 & 67 \\
El Salvador & 1,1 & 92 \\
Guatemala & 1,4 & 90 \\
Honduras & 1,6 & 88 \\
México & 2,0 & 80 \\
Nicaragua & 0,8 & 91 \\
Panamá & 2,9 & 44 \\
República Dominicana & 0,4 & 50 \\
Media & 1,6 & 75 \\
\hline
\end{tabular}

Fuente: elaboración de los autores, con base en la referencia 19.

pagaba US $\$ 0,85^{5}$ por una dosis diaria de tratamiento antipsicótico, lo que representaba $19 \%$ del salario mínimo diario en México. En el caso de los antidepresivos, el costo de la dosis diaria era de US\$ 0,17 y equivalía a $4 \%$ del salario mínimo diario.

\section{Servicios de salud mental, atención primaria y recursos humanos}

Se observó que el eje de la atención de salud mental era -y sigue siendo- el hospital psiquiátrico. Un buen ejemplo de tal afirmación es que, al momento del estudio, existían 46 hospitales psiquiátricos frente a 13 unidades de internamiento psiquiátrico en hospitales generales y 8 en establecimientos residenciales. Aunado a lo anterior, el acceso se veía limitado por barreras geográficas, ya que la mayoría de los servicios se encontraba en las grandes metrópolis o sus alrededores. Si bien es cierto que el número de servicios ambulatorios fue más amplio, no alcanzaba para cubrir las necesidades de atención y también se registró una cantidad insuficiente de servicios enfocados a la atención de los niños y adolescentes. Solo $27 \%$ del total de usuarios atendidos en establecimientos ambulatorios eran niños o adolescentes, y ese porcentaje caía a $6 \%$ en los hospitales psiquiátricos. Los resultados también revelaron que aproximadamente 50\% de los pacientes que recibían atención lo hacían en servicios hospitalarios y que era internado un paciente de cada 1,7 atendido en los servicios ambulatorios.

Los usuarios de los servicios ambulatorios informaron un promedio de

\footnotetext{
5 El tipo de cambio en 2008 era de US $\$ 1,00=10,50$ pesos mexicanos.
}

cuatro contactos por año, 31\% de los establecimientos proporcionaba atención de seguimiento en la comunidad y $4 \%$ contaba con equipos móviles. Todos o casi todos los servicios (81-100\%) habían realizado una o más intervenciones psicosociales durante el año anterior al estudio y $67 \%$ tuvieron disponible por lo menos un medicamento psicotrópico de cada clase terapéutica (antipsicóticos, antidepresivos, estabilizadores del estado de ánimo, ansiolíticos y antiepilépticos). Por su parte, $80 \%$ de los hospitales psiquiátricos había llevado a cabo algún tipo de intervención psicosocial durante el año anterior al estudio y, de ellos, 98\% dispuso de medicamentos psicotrópicos. En promedio, los pacientes estuvieron hospitalizados menos de un mes (24 días), pero $38 \%$ había permanecido un período mucho más elevado (cinco o más años en hospitales psiquiátricos).

$\mathrm{Al}$ interior de las prisiones se registró un número reducido de camas destinadas a los reclusos con trastornos mentales, mas en ninguna de ellas existía un servicio específico de salud mental. No más de $30 \%$ de los establecimientos de atención primaria contaba con protocolos de evaluación y tratamiento para condiciones clave de salud mental y realizaba - como mínimo- una referencia al mes con un especialista en salud mental. Del mismo modo, el porcentaje de cursos de actualización y/o de educación relacionados con temas de salud mental que estaban dirigidos a los profesionales que trabajan en este nivel de atención fue menor a $15 \%$.

En México, como en muchos otros países de América Latina (24), los recursos humanos enfocados a la salud mental informaron niveles insuficientes $\mathrm{y}$, en la mayoría de los casos, estaban concentrados en los hospitales psiquiátricos (figura 1).

\section{Educación sanitaria y vínculos con otros sectores}

Es importante mencionar que se registró una vinculación escasa entre el sistema de salud mental y la sociedad civil. Tanto es así, que no se cuenta con información sistematizada sobre las asociaciones de usuarios y familiares. Con base en la poca información existente, se estimó que solamente $20 \%$ de este tipo de asociaciones interactúa de forma regular con servicios de salud mental y que un porcentaje similar ha participado alguna vez en la formulación e implementación de políticas, planes o normativas sobre salud mental. Asimismo, los programas destinados a que las personas con problemas mentales tengan acceso a un trabajo remunerado y a otras prestaciones sociales registraron un número muy bajo, a pesar de existir disposiciones legislativas relacionadas con el empleo, la protección contra la discriminación y el aseguramiento de la vivienda.

\section{Monitoreo e investigación}

El trabajo de monitoreo incluye la elaboración de informes periódicos con indicadores básicos de los diferentes establecimientos de salud mental (número de camas, admisiones, admisiones involuntarias, duración de la estadía, diagnóstico de los pacientes). Durante 2008 la Secretaría de Salud recibió este tipo de datos de $87 \%$ de los hospitales psiquiátricos, $54 \%$ de las unidades psiquiátricas 
FIGURA 1. Distribución de los recursos humanos en salud mental (tasa por 100000 habitantes) en Brasil, Chile, Costa Rica y México, 2005-2009

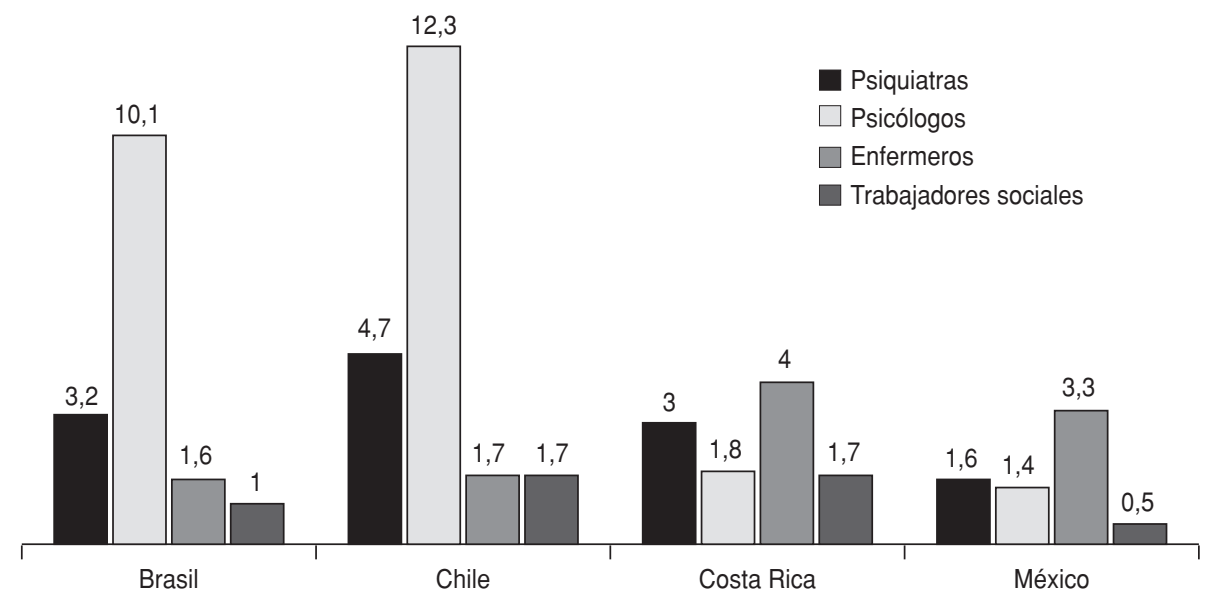

Fuente: elaboración de los autores, con base en la referencia 24.

en hospitales generales y $60 \%$ de los establecimientos ambulatorios.

Con respecto a la investigación, en México se realiza un trabajo continuo y sistemático que aborda diversos aspectos de salud mental, como estudios epidemiológicos, evaluaciones clínicas, intervenciones psicosociales y psicoterapéuticas, y estudios genéticos. En este sentido, y de acuerdo a la revisión realizada en la base de datos PubMed, en 2008 se publicaron en el país 1449 artículos relacionados con la salud, de los cuales $26 \%$ correspondió a estudios sobre salud mental.

\section{DISCUSIÓN}

Como lo muestran los datos presentados, el eje de la atención de la salud mental en México sigue siendo el hospital psiquiátrico, a pesar de que las últimas políticas y acciones mundiales de salud mental ubican a la atención primaria como el punto desde donde articular la atención de la salud mental comunitaria (25).

Esta gran concentración de la atención en los hospitales psiquiátricos ha dado lugar a que una alta proporción de pacientes sean internados en dichos establecimientos, en lugar de realizar un tratamiento ambulatorio - -la relación es que se interna 1 de cada 1,7 pacientes que reciben servicios ambulatorios. Este desequilibrio demuestra que la atención de los trastornos mentales no está del todo integrada en el sistema nacional de salud y que hace falta un mecanismo adecuado de detección y tratamiento en los primeros niveles, así como un efectivo sistema de referencia.

Otro aspecto de suma importancia es la constatación de los escasos servicios orientados a la atención de niños y adolescentes, lo que resulta muy preocupante puesto que es precisamente durante las primeras décadas de la vida cuando comienzan la mayoría de los trastornos psiquiátricos (26).

A partir de expuesto, se torna perentorio reflexionar acerca de un nuevo rumbo para la atención de la salud mental en México. Con tal propósito, se exponen a continuación algunas propuestas:

1. Aumentar de manera paulatina la proporción de gasto en salud destinado a salud mental y reestructurar el modo de distribución del presupuesto, lo que ayudaría a disminuir la brecha entre la carga que representan los trastornos mentales y el presupuesto asignado para su atención.

2. Ubicar a los servicios de atención primaria como ejes articuladores de la atención en salud mental. Para ello, será necesario fortalecer los equipos y programas de salud mental en el primer nivel de atención, así como mantener una adecuada coordinación entre los diferentes niveles.

3. Transformar a los hospitales psiquiátricos en unidades de atención especializada, con las camas necesarias para atender solamente a las personas que requieran hospitalizaciones prolongadas (27).

4. Fortalecer la enseñanza de la salud mental en el pregrado de medicina y en las carreras de psicología, enfermería y trabajo social, entre otras, y mantener una capacitación continua - sobre salud mental- en los primeros niveles de atención.

5. Incrementar la participación de la sociedad civil y establecer vínculos sólidos entre el sector salud y las asociaciones de usuarios y familiares, de manera que tengan una participación más proactiva en la planificación y desarrollo de los programas y servicios de salud mental.

\section{Alcances y limitaciones}

El uso del IESM-OMS ha permitido realizar una evaluación multidimensional del sistema de salud mental mexicano. Gracias a ello, se dispone de información actual sobre las características y necesidades de los servicios de salud mental, la que puede resultar muy útil a la hora de diseñar e implementar políticas públicas como reformas normativas o planes y programas de acción específicos.

Adicionalmente, también es posible comparar estos resultados con los de otros países, ya que los criterios del instrumento utilizado han sido estandarizados. En tal sentido, el IESM-OMS se constituye como una gran oportunidad para homogeneizar los criterios de recolección 
de datos, lo que contribuirá significativamente a la construcción de un sistema nacional y uno regional de información en salud mental basados en datos confiables.

Las limitaciones del instrumento incluyen una falta de indicadores sobre las características sociales, políticas y culturales de cada país, como ya han destacado otros autores (20). La conjugación de esas condiciones suele determinar buena parte de las ideas sobre el proceso salud-enfermedad de la población, sus necesidades y la búsqueda de atención, lo que adquiere aún mayor relevancia si se considera la gran diversidad cultural que existe entre los pueblos de la Región.

Por último, sería conveniente contar con herramientas teórico-metodológicas que permitieran conocer cómo se articulan las prácticas de atención no convencionales con el sistema de salud mental formal, puesto que esas relaciones podrían constituir una de las fortalezas no previstas para la formulación de políticas públicas de salud. Más aún, es importante señalar que si las estrategias informales utilizadas por la población para cuidar la salud mental fuesen omitidas del análisis, se correría el riesgo de obtener un subregistro, tanto de los trastornos mentales como de los actores sociales implicados en el proceso de salud-atención.

Conflictos de interés. Ninguno declarado por los autores.

\section{REFERENCIAS}

1. World Health Organization. Mental Health Atlas 2011. Geneva: WHO; 2012.

2. Rodríguez JJ, Barrett T, Narváez S, Caldas JM, Levav I, Saxena. Sistemas de salud mental en el Salvador, Guatemala y Nicaragua: resultados de una evaluación mediante el WHO-AIMS. Rev Panam Salud Publica. 2007;22(5):348-57.

3. World Health Organization. World Health Organization Assessment Instrument for Mental Health Systems. Version 2.2. Geneva: WHO; 2005

4. Saxena S, Lora A, van Ommeren M, Barrett T, Morris I, Saraceno B. WHO's Assessment Instrument for Mental Health Systems: collecting essential information for policy and service delivery. Psychiatr Serv. 2007;58(6)816-21.

5. México, Instituto Nacional de Estadística y Geografía (INEGI). Censo de Población y Vivienda 2010. México: INEGI; 2011.

6. México, Consejo Nacional de Evaluación de la Política de Desarrollo Social (CONEVAL). Informe de Pobreza Multidimensional en México, 2008. Disponible en: http:// www.coneval.gob. $\mathrm{mx} / \mathrm{cmsconeval/}$ $\mathrm{rw} /$ resource/coneval/med_pobreza / Informe_pobreza_multidimensional/ Informe de Pobreza Multidimensio nal_en_Mexico_2008_.pdf Acceso el 19 de marzo de 2013.

7. Almeida C. Reforma de sistemas de servicios de salud y equidad en América Latina y el Caribe: algunas lecciones de los años 80 y 90 . Cad Saude Publica. 2002;18(4):905-25.

8. México, Secretaría de Salud. Catálogo Universal de Servicios de Salud (CAUSES), 2010. Disponible en: http://www.isea.gob.mx/ Formatos/CAUSES_2010.pdf Acceso el 19 de marzo de 2013.

9. Homedes N, Ugalde A. Las reformas de salud neoliberales en América Latina: una visión crítica a través de dos estudios de caso. Rev Panam Salud Publica. 2005;17(3):210-20.

10. López O, Blanco J. La polarización de la política de salud en México. Cad Saude Publica. 2001;17(1):43-54.
11. González-Pier E, Gutierrez C, Stevens G, Barraza M, Porras R, Caravalho N, et al. Priority setting for health interventions in Mexico's system of social protection in health. The Lancet. 2006;368(9547):1608-18.

12. Patel V. Mental health in low- and middleincome countries. Br Med Bull. 2007;8182(1):81-96.

13. Kohn R, Rodríguez J. Prevalencia y carga de los trastornos mentales en la población adulta de América Latina y el Caribe. En: Rodríguez J, Kohn R, Aguilar S, eds. Epidemiología de los trastornos mentales en América Latina y el Caribe. Washington DC: OPS; 2009. Pp. 19-32.

14. Medina-Mora ME, Borges G, Lara C, Benjet C, Blanco J, Fleiz C, et al. Prevalencia de trastornos mentales y uso de servicios: Resultados de la Encuesta Nacional de Epidemiología Psiquiátrica en México. Salud Ment (Mex). 2003;26(4):1-16.

15. Medina-Mora ME, Borges G, Lara $C$, Benjet C, Blanco J, Fleiz C, et al. Prevalence, service use, and demographic correlates of 12-month DSM-IV psychiatric disorders in Mexico: results from the Mexican National Comorbidity Survey. Psychol Med. 2005;35:1-11.

16. Borges G, Medina-Mora ME, Wang PS, Lara C, Berglund P, Walters E. Treatment and adequacy on treatment of mental disorders among respondents to the Mexico National Comorbidity Survey. Am J Psychiatry. 2006;163(8):1371-8

17. Borges G, Wang PS, Medina-Mora ME, Lara C, Chiu WT. Delay of first treatment of mental and substance use disorders in Mexico. Am J Public Health. 2007;97(9):1638-43.

18. Kohn R, Levav I, Caldas de Almeida JM, Vicente B, Andrade L, Caraveo-Anduaga JJ, et al. Los trastornos mentales en América Latina y el Caribe: asunto prioritario para la salud pública. Rev Panam Salud Publica. 2005;18(4/5):229-40.

19. Aparicio V, Saxena S, Rodríguez J. Evaluación de los Sistemas de Salud Mental en Centroamérica y la República Dominicana. En: Rodriguez J, Kohn R, Aguilar S, eds. Epide- miología de los trastornos mentales en América Latina y el Caribe. Washington DC: OPS; 2009. Pp. 289-99.

20. Organización Mundial de la Salud. Informe sobre la salud en el mundo, 2001. Salud mental: Nuevos conocimientos, nuevas esperanzas. Disponible en: http://www.who.int/ whr/2001/es/index.html Acceso el 19 de marzo de 2013.

21. Hamid H, Abanilla K, Bauta B, Huang KY. Evaluating the WHO Assessment Instrument for Mental Health Systems by comparing mental health policies in four countries. Bull World Health Organ. 2008;86(6):467-73.

22. Jones J, Hunter D. Consensus methods for medical and health services research. BMJ. 1995;311(7001):376-80.

23. World Health Organization. Assessment Instrument for Mental Health Systems version 2.2. Disponible en: http://www.who.int/ mental health/evidence/AIMS_WHO_2 2. pdf Acceso el 19 de marzo de 2013.

24. World Health Organization. Mental health. WHO-AIMS Country Reports. Disponible en: http://www.who.int/mental health/ who_aims_country_reports/en/index. html Acceso el 8 de abril de 2013.

25. Organización Mundial de la Salud. Programa de acción para superar las brechas en salud mental. Ginebra: OMS; 2008.

26. Benjet C, Borges G, Blanco J, Rojas E, Fleiz E, Méndez E, et al. La Encuesta de Salud Mental en Adolescentes de México. En: Rodríguez J, Kohn R, Aguilar-Gaxiola S, eds. Epidemiología de los trastornos mentales en América Latina y el Caribe. Washington DC: OPS; 2009. Pp. 90-100.

27. Organización Panamericana de la Salud (OPS). Evaluación de Servicios de Salud Mental en la República Mexicana. Washington, D.C.: OPS; 2004.

Manuscrito recibido el 2 de mayo de 2012. Aceptado para publicación, tras revisión, el 30 de noviembre de 2012. 
ABSTRACT Objective. Evaluate some of the key indicators that characterize the Mexican mental health system using the World Health Organization's Assessment Instrument for Mental Health Systems (WHO-AIMS).

\section{Evaluation of the mental health system in Mexico: where is it headed?}

Methods. The strategy for examining the WHO-AIMS indicators included: (i) a review of documentary sources; (ii) application of the questionnaire; and (iii) group work with a team of experts using the consensus technique. To facilitate collection of the data, a questionnaire was prepared in which the indicators were turned into simple questions. The people gathering the data were trained, and the activity was monitored.

Results. It was found that, of the total budget for health, only $2 \%$ is allocated for mental health, and, of that share, $80 \%$ is used in the operation of psychiatric hospitals. The pivotal point for mental health care is in the psychiatric hospital; there are very few psychiatric units in the general hospitals, few residential establishments, and few services targeted specifically to care for children and adolescents. Access is limited because of the centralized health care system, with the majority of establishments located in the large cities. Only 30\% of primary care services have protocols for the evaluation and treatment of mental disorders. Finally, in the mental health facilities, the ratios of psychiatrists, other physicians, nurses, and psychologists per 100000 population are 1.6, 1.3, 3.4, and 1.5, respectively.

Conclusions. More funding will be needed in order to bridge the gap between the mental health burden and the budget allocated for its care, and resources will need to be used more rationally, with the first level of care becoming the pivot. In addition, it will be necessary to increase the number of specialists, offer periodic in-service training for personnel at the first level of care, and enlist greater participation by the rest of society.

Key words Mental health; policy making; hospitals, psychiatric; mental disorders; mental health services; Mexico. 